\title{
Miranda
}

Revue pluridisciplinaire du monde anglophone /

Multidisciplinary peer-reviewed journal on the English-

speaking world

$8 \mid 2013$

In Umbra Voluptatis : Shades, Shadows, and their

Felicities / Film Adaptations, New Interactions

\section{Adapter le 11 septembre 2001 au cinéma : Limites et écarts de la représentation dans World Trade Center (Oliver Stone, 2006)}

\section{Vincent Souladié}

\section{(2) OpenEdition}

12 Journals

Édition électronique

URL : http://journals.openedition.org/miranda/3556

DOI : 10.4000/miranda.3556

ISSN : 2108-6559

Éditeur

Université Toulouse - Jean Jaurès

Référence électronique

Vincent Souladié, «Adapter le 11 septembre 2001 au cinéma : Limites et écarts de la représentation dans World Trade Center (Oliver Stone, 2006) », Miranda [En ligne], 8 | 2013, mis en ligne le 28 juin 2013, consulté le 16 février 2021. URL : http://journals.openedition.org/miranda/3556 ; DOI : https://doi.org/ 10.4000/miranda.3556

Ce document a été généré automatiquement le 16 février 2021.

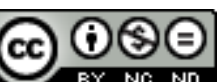

Miranda is licensed under a Creative Commons Attribution-NonCommercial-NoDerivatives 4.0 International License. 


\title{
Adapter le 11 septembre 2001 au cinéma : Limites et écarts de la représentation dans World Trade Center (Oliver Stone, 2006)
}

\author{
Vincent Souladié
}

\section{Introduction}

1 Cet article propose d'analyser les implications esthétiques de l'adaptation cinématographique des attentats du 11 septembre 2001, envisagés comme événement surmédiatisé. Comment le cinéma hollywoodien donne-t-il à voir et à penser la catastrophe, quelle considération accorde-t-il à son identité visuelle préétablie, avec quelle part d'autonomie formelle s'empare-t-il de sa reconstitution? En somme, cette dernière se tient-elle à distance de ce que la représentation médiatique nous a enseigné ou réinvestit-elle ses tropes figuratifs les plus fameux? Le terme même d'adaptation supposerait que l'événement soit un fait homogène sujet à une seule interprétation possible. Or, l'événement étant par définition polysémique, nous sommes ici conduit à interroger l'objet référentiel sur lequel s'appuie la transposition filmique. La problématique de l'adaptation hollywoodienne repose selon nous à la croisée de ces deux interrogations concurrentes : comment représenter l'événement si médiatisé du 11 septembre 2001 sans trahir la mémoire scopique des spectateurs et « comment représenter les événements (...) sans pour autant alimenter le spectacle qui les a déjà mis en forme?" (Gervais). Nos réflexions porteront exclusivement sur World Trade Center (Oliver Stone, 2006), second long métrage à avoir tenté le défi de la reconstitution du $11 / 9^{1}$ et, à ce jour, seul film de fiction centré sur son épisode le plus visible : les attentats de Manhattan et la destruction des tours jumelles.

2 Une précision lexicale tout d'abord. Après les attentats de septembre 2001, le discours des commentateurs publics a eu pour charge de cristalliser l'événement autour d'une 
représentation collective et fédératrice, permettant au lecteur de le saisir, c'est-à-dire de pouvoir en parler. Aussi les médias et le public américains ont-ils communément adopté l'expression «9/11», rentrée dans le langage courant. Jacques Derrida remarque la tentation, commune à nombre d'observateurs au lendemain des attentats, de réduire la tragédie du onze septembre à son seul climax spectaculaire, réflexe qui ressort selon lui d'une dénégation de la réalité dramatique en tant que phénomène inqualifiable et inexplicable. Le simple fait de nommer l'événement par la date de sa réalisation serait, selon Derrida, l'aveu d'un échec de sens. «L'index pointé vers cette date, l'acte nu, le déictique minimal, la visée minimaliste de cette datation marque [...] qu'on ne dispose peut-être d'aucun concept et d'aucun sens pour nommer autrement cette «chose » qui vient d'arriver, ce supposé «événement » (Borradori, 134). Pour traduire en français « 9/11» en respectant sa concision, nous prenons alors le parti pris de choisir l'équivalent "11/9", plutôt que " 11 septembre » en toutes lettres. Plus qu'une simple date historique, le terme $9 / 11$, ou pour nous $11 / 9$, se fait l'emblème du désarroi provoqué par un acte violent et imprévisible, inédit et asémantique, porteur d'un logos intraduisible.

3 Pour avoir transi quasi simultanément une incommensurable communauté de regards, les attentats commis sur le sol américain le 11 septembre 2001 sont décrits par Jürgen Habermas comme le «premier événement historique mondial» (Borradori 57). Selon ce dernier, la catastrophe devrait cette participation spectatorielle inédite à « la présence des caméras et des médias qui a fait d'un événement local un événement planétaire la population du monde entier devenant un témoin oculaire médusé » (Borradori, 58). Cette participation scopique plurielle, assortie des propriétés spectaculaires des images de l'acte terroriste, a tôt fait d'ouvrir des analogies avec le cinéma catastrophe, immense pourvoyeur de fictions d'apocalypse urbaine ${ }^{2}$. L'opprobre a ainsi rapidement été jetée sur ce genre cinématographique, accusé d'avoir inspiré les commanditaires de l'attentat ${ }^{3}$, d'avoir déréalisé toute catastrophe et de ne pas nous avoir préparés à son surgissement dans la réalité ${ }^{4}$, ou désigné encore comme mètre-étalon de nos fantasmes apocalyptiques auxquels les images médiatiques ont dû se mesurer au point de déréaliser l'événement lui-même ${ }^{5}$. A notre sens, ces réflexions ont pour point commun primordial de ne retenir de l'attentat que sa visibilité médiatique mais elles n'interrogent ni les conditions de son exécution ${ }^{6} \mathrm{ni}$ la distance des images.

4 La production hollywoodienne, directement visée par ces invectives, subit en deuxième instance un autre procès d'intention lorsqu'on soupçonna qu'elle puisse chercher à transposer la catastrophe sur grand écran. Claude Lanzmann fut en France l'un des premiers à exprimer ses craintes à cet égard :

C'est une idée qui me paraît complètement folle [de reconstituer au cinéma les attentats du 11/9]. Rien ne sera aussi puissant que ces images réelles, que nous avons vues. Ces images volées à la réalité sont d'une extraordinaire qualité cinématographique, et elles ont eu immédiatement un public planétaire, fasciné par l'horreur, public que nul Spielberg n'atteindra jamais. Après des documents pareils, quel est le metteur en scène assez fou pour se dire: je peux faire mieux? (Lanzmann 56).

Retenons de cette réflexion deux idées essentielles afin de mieux saisir le type d'opposition auquel a dû se heurter le cinéma Hollywoodien lorsque se concrétisa le projet de porter à l'écran le 11/9. Tout d'abord, les films se seraient vu concurrencés à jamais par des images de la réalité qui excèderaient leur propre pouvoir esthétique. Ensuite, le désir de l'industrie cinématographique américaine d'en découdre avec un 
drame collectif aussi amplement partagé ne serait satisfait qu'au nom d'une orgueilleuse visée compétitive tant artistique que commerciale: surpasser l'image indicielle de la réalité et fédérer une audience massive devant le simulacre fictionnel. L'enjeu futile de l'adaptation du 11/9 serait alors de compenser le déclassement des images filmiques et de faire reconquérir au cinéma son ancien pouvoir.

Mais l'allégation que formule ici Claude Lanzmann ne préjuge-t-elle pas des potentialités et des limites du cinéma américain industriel, envisagé comme une instance monstratoire impérieuse ? Concurrencé par la réalité d'une catastrophe dont la présentation télévisée phagocyte ses tropes figuratifs les plus fameux, le cinéma hollywoodien n'aurait-il d'autres ressources que la surenchère spectaculaire ? Telle fut l'une des craintes exprimées par de nombreux spectateurs après le $11 / 9$, dont les propos cités ci-dessus se font l'écho. Quoique très prévisible, le basculement de l'événement historique dans la fiction ne laissait pourtant pas augurer du traitement esthétique qu'il allait recevoir. L'enjeu de l'adaptation cinématographique du 11/9, comme de toute reconstitution, ne doit-elle pas venir de la distance que celle-ci instaure avec l'événement lui-même plutôt que d'une inatteignable homologie? Telle est la position que tient notamment Priska Morrissey lorsqu'elle avance que « le hiatus de la reconstitution consiste dans l'écart entre le fantasme de la résurrection propre au cinéma comme à l'Histoire et l'impossibilité intrinsèque de cette résurrection " (Morrissey, 112).

\section{Mondialisation de l'image et intégrité artistique}

7 De par son sujet, World Trade Center est confronté à l'excessive médiatisation de l'événement, décrite plus haut par Habermas. En effet, la perception de la catastrophe s'est un temps réduite à une série d'images identiques sempiternellement rediffusées par les chaînes de télévision et publiées dans la presse écrite ${ }^{7}$. Face à cette emprise iconographique, au moins deux positions s'affrontent. La première considère la couverture télévisée de l'événement comme facteur utile de cohésion sociale. Jocelyne Arquembourg soutient par exemple que la répétition de ces images traumatiques était nécessaire pour donner forme à l'événement, pour en délimiter les bornes, et pour ainsi permettre la célébration du deuil collectif : « les médias deviennent ainsi le lieu d'une célébration qui assigne au public un rôle participatif sur le mode de la communion " (Arquembourg, 84).

8 A l'opposé, un autre courant d'idées met en garde contre une prolifération dangereuse des images devant lesquelles le spectateur se retrouverait aveuglément prisonnier. Olivier Mongin et Paul Virilio figurent peut-être parmi les plus virulents dénonciateurs français de ce pouvoir. Le premier proclame que l'image télévisée est devenue « autoréférentielle [...] puisqu'elle ne fait rien d'autre que renvoyer à d'autres écrans en permanence » (Mongin, 220). Paul Virilio s'inquiète quant à lui du fait que « sans aucun recul critique devant la déferlante, le spectateur est ainsi soumis à [...] l'hallucination collective d'une image unique » qui contribue à ce qu'il nomme un "bouclage de l'imaginaire»(Virilio, 90). Ces lectures contradictoires des images trouvent pour terrain d'entente l'idée que la représentation collective de l'événement repose sur une faible disparité iconographique. Ainsi, le régime de la survisibilité médiatique a arbitrairement contribué selon nous à imposer une représentation collective uniforme et souveraine de l'événement. Cinq ans après les attentats, comment World Trade Center 
se comporte-t-il dans le faisceau de représentations orchestré par la mondialisation de l'image?

9 L'un des enjeux esthétique posé par l'adaptation semble se situer autour de la question de l'appropriation artistique. Si les images médiatiques formatent la représentation de l'événement pour lui donner le visage d'un fait objectif, la reconstitution cinématographique est a priori en mesure de leur substituer le point de vue du cinéaste et de son regard potentiellement surplombant, déformant ou biaisé, mais dans tous les cas, autre.

Bien qu'Oliver Stone soit un réalisateur attaché à New York $^{8}$, sa présence derrière la caméra de World Trade Center fit à ce titre question. Cinéaste engagé (Platoon, Né Un Quatre juillet), volontiers provocateur, habitué aux controverses, célèbre pour l'outrance formelle et la violence graphique de certains de ses films (Tueurs Nés, Alexandre), soucieux de mettre en image les vérités souterraines qui hantent la nation américaine (JFK, Nixon), Oliver Stone ne semble pas disposer a priori du profil le plus approprié pour s'emparer d'un sujet aussi fragile avec retenue. Stephen Prince note que dans les années 80 , Stone se trouve être «le seul cinéaste à réaliser des films populaires qui critiquent à la fois la politique extérieure et intérieure des Etats Unis » (Prince, 2000, 260). D'ailleurs, comme le rappelle Robert Brent Toplin, Stone a l'habitude de revendiquer ouvertement sa position de cinéaste attaché à imposer sa

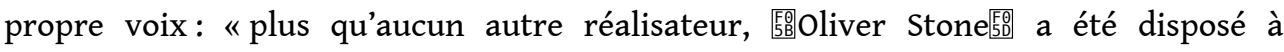
participer à des débats publics à propos des aspects controversés de ses films » (Toplin, p.4). Or, Stone, qui n'avait auparavant jamais travaillé avec la Paramount, société productrice du film, s'entendit expressément demander de ne pas faire montre dans World Trade Center d'un quelconque discours frondeur. Le cinéaste fut choisi par la production à la seule condition de gommer ce qui fait l'une de ses spécificités artistiques, ou tout au moins sa réputation d'auteur.

Auprès du public et de la critique, le choix de ce réalisateur par la Paramount s'est d'abord prêté à la défiance et à l'incompréhension. Avant la sortie de World Trade Center, l'horizon d'attente du public semblait nourri de la notoriété d'Oliver Stone, de son engagement politique revendiqué et de l'ambition affichée par son œuvre d'embrasser sans concession l'Histoire américaine contemporaine et ses plus violents soubresauts. Mais comme le rappelle Stephen Prince, "Stone a surpris tout le monde en faisant un film qui mettait en valeur l'héroïsme et l'élévation morale et ne comportait aucun point de vue social ou politique sur les attentats ${ }^{9}$ (Prince, 2009, 100). De même, pour Daniel Mendelsohn :

Ce n'est pas le genre de film que l'on attend d'oliver Stone, qui a un faible pour l'épopée, voire le théâtralisme et qui, dans des films comme Né un 4 juillet, JFK et Nixon, s'est régalé à exaspérer le public en réinterprétant l'histoire politique récente avec une bonne dose de cette paranoïa que l'on associe souvent aux extrémistes de gauche. [...] C'est pourquoi, avant la sortie du film, tout le monde s'attendait à ce que World Trade Center traite ce jour épique de manière grandiose (Mendhelson, 299).

12 En somme, la personnalité artistique d'Oliver Stone semblait le disqualifier d'emblée et pouvait laisser craindre un manque de subtilité jugé nuisible. Pourtant, encore une fois, cette méfiance préventive ne doit pas faire oublier, ainsi que le défend François Jost, que "chaque spectateur a un auteur dans un coin de sa tête, somme hétéroclite d'informations reçues, de souvenirs de films déjà vus, de fantasmes, en bref, un auteur construit » (Jost 53). Une vue générale du film nous permet de constater immédiatement 
que Stone ne renoue pas ici avec le style «hyperexpressif» (Berthomieu 506) qui a caractérisé une part de son cinéma de Nixon (1995) à Alexandre (2004), et qui a pu prêter le flanc aux critiques hésitantes à l'annonce de sa participation à World Trade Center. Le cinéaste abandonne pour un temps la forme du montage dialectique et hystérique grâce auquel «la syntaxe de ses films est un rude exercice mental pour le spectateur» (Berthomieu 506-507). Comment Oliver Stone s'approprie-t-il alors l'événement pour en construire l'adaptation? Le cinéaste reste-t-il dans les rails d'une représentation préétablie? L'événement médiatique possède-t-il une autorité hiératique qui influe sur l'approche du cinéaste jusqu'à lui faire sacrifier certaines de ses spécificités esthétiques dominantes? Ou bien, inversement, Oliver Stone déchire-t-il l'écrin du visible par le réinvestissement de certaines figures récurrentes de son œuvre?

World Trade Center narre le calvaire de John McLoughlin (Nicolas Cage) et Will Jimeno (Michael Peña), deux officiers du Port Authority Police Department de Manhattan, reconvertis en secouristes le matin du 11/9. Après la chute des tours, ils se trouvèrent prisonniers douze heures durant sous six mètres d'acier et de gravats, dans un minuscule et obscur espace confiné en partie épargné par le choc, immobiles et sans moyens de communication avec l'extérieur. Pour mesurer les implications formelles de l'adaptation du $11 / 9$, notre intérêt se portera principalement sur quatre séquences clés, toutes situées dans les vingt premières minutes du film, avant l'effondrement des tours du World Trade Center.

\section{Sérénité mensongère}

Le film débute à l'aube, le matin du 11 septembre 2001, dans les quartiers péri-urbains de New York. Le vétéran John McLoughlin et le novice Will Jimeno quittent leur domicile respectif en direction de Manhattan pour prendre leur service. Les petits gestes matinaux ordinaires des personnages sont alternés par le montage avec des plans d'ensemble extérieurs fixes et descriptifs dans les décors tranquilles des quartiers résidentiels populaires, artères quasi désertes, ponts, toits et façades des immeubles éteints, camions de marchandises béants, glacés dans la teinte monochrome bleu-nuit appliquée à l'image par le directeur de la photographie Seamus McGarvey pour simuler l'épaisseur de l'obscurité nocturne qui lentement et calmement s'estompe. Contemplatif plutôt que dynamique, cet enchaînement de plans gèle une quasi-stase transitoire insoumise à toute direction dramaturgique, comme illustrée par cette forme de résistance chromatique de la nuit sur le jour. Les notes claires et espacées de la mélodie de piano composée par Craig Armstrong ajoutent à la quiétude et à la lenteur de cette ouverture filmique. Pourtant, dans le décor pointe en plusieurs occasions un élément figuratif de nature à faire frémir la sérénité descriptive.

En effet, tout au long de cette énumération spatiale, par trois fois et depuis trois points de vue différents, la caméra fixe les tours du World Trade Center en profondeur de champ au centre du cadre et du paysage urbain. Les tours jumelles que nous voyons ne sont en aucun cas présence indicielle du bâtiment dans une image du passé, mais reconstitution numérique dans une image actuelle. Le spectateur n'est bien sûr pas explicitement informé de la nature profilmique du matériau iconique, comment le serait-il à moins d'un effet spécial imparfait ou défectueux sapant indubitablement l'effet de réalité ? Dès lors, nous pouvons nous demander pourquoi des images d'archives, par exemple, n'ont pas été employées, éventuellement maquillées pour les 
raccorder chromatiquement aux plans précédents, et intégrées incognito à la chaine filmique? Posons l'hypothèse qu'Oliver Stone a eu recours aux effets numériques, pourtant plus dispendieux, pour constituer une image de New York qui ne doive rien à une iconographie trop facilement perçue comme surannée. Celle-ci ne doit aucunement référer au présent du tournage mais paraître dépendante figurativement du présent diégétique.

La première apparition a lieu lors du cinquième plan de la séquence, lequel attire notre attention. La large rivière Hudson trace une ligne de fuite vers les deux fines silhouettes verticales érigées en fond, serrées sur la bande d'horizon couleur abricot entre les masses étales de la rivière et des nuages. Cet encadrement naturel intensifie leur discrète mais entêtante présence. Sans doute eut-il été déroutant de la part d'Oliver Stone de ne pas présenter d'emblée l'édifice iconisé qui donne son titre au film. Plutôt que de faire attendre son entrée et de lui réserver un surgissement-surprise dans le cadre, Stone le rejette dans le lointain de l'arrière-plan, comme point de convergence scopique et spatial. Si le lieu choisi pour la prise de vue est assez peu commun au cinéma, ce qui en fait sa nouveauté paradoxale, il pourrait renvoyer en revanche à l'école picturale de l'Hudson River School ${ }^{10}$ qui fit de la nature bucolique et luxuriante de l'Amérique un modèle. Par ce recours possible à la composition de l'imagerie primitive, Stone semble renouer avec la scène new-yorkaise originaire, il s'applique à la restauration d'un paysage qui n'est plus et que l'on contemple depuis une manière de mémoire picturale où le mythe prend corps.

Plus loin dans la séquence, les tours sont vues depuis le ferry remontant la rivière vers Manhattan. Alors qu'un personnage secondaire s'avance et s'appuie contre la proue, un panoramique le suit dans son déplacement jusqu'à laisser entrer l'édifice du World Trade Center dans le cadre. Cette fois-ci, l'irruption s'est produite en conclusion d'un mouvement de caméra aménageant un jeu de dévoilement avec la figure. Encore plus loin, en terminaison de l'incipit, nous accompagnons le véhicule de Will Jimeno traverser le pont de Williamsburg. La caméra s'élève en pano-travelling et cadre, de l'autre côté de la rive, la silhouette si caractéristique des gratte-ciels de la ville encore une fois dominés par les tours jumelles. Au bas de l'écran se superpose alors un bandeau mentionnant en fines lettres capitales blanches la date de l'action: «September 112001 ». Le mouvement de caméra agit à notre sens en tant qu'instance énonciative ${ }^{11}$, en guidant notre regard il assure la rencontre entre la figure humaine et la figure architecturale et la dramatise. Par cette liaison, le paysage est alors converti en lieu diégétique et siège du récit. Nous pouvons supposer qu'avec cette séquence d'ouverture, Oliver Stone donne lentement corps à la narration plutôt que de lancer d'entrée le spectateur dans l'urgence de l'action.

Certes, un tel incipit n'est rien moins que stéréotypé si l'on considère que nombre de films situés à New York commencent conventionnellement par des vues d'ensemble du paysage de la ville ${ }^{12}$. Pourtant, plutôt que de la réduire seulement ici à une forme d'académisme générique, il nous paraît également pertinent d'envisager cette ouverture en tant que repère esthétique dans l'œuvre même d'oliver Stone. En effet, c'est la troisième fois que ce dernier ouvre un de ses films par une séquence descriptive de l'aube se levant sur New York ${ }^{13}$. Dans Last Year in Vietnam (1971), son film de fin d'études tourné en noir et blanc, Stone se met en scène dans la peau d'un vétéran du Vietnam redécouvrant New York après sa démobilisation. Dans les premiers plans descriptifs du film, la lumière blanche du soleil levant y saisit crûment la silhouette 
spectrale des gratte-ciels. En parallèle, des plans tournés en couleur représentent une nature verdoyante figurant les paysages du Vietnam, réminiscence romantique de l'ailleurs qui contraste avec la solitude et le désenchantement du retour au pays du vétéran estropié ${ }^{14}$. Sans que le souvenir chromatique du Vietnam soit explicitement lié à l'idée de traumatisme, le noir et blanc de la ville dans laquelle déambule le héros tout au long du film semble marquer l'impossibilité d'habiter le présent.

Plus tard, dans Wall Street (1987), Stone ouvre son film en une série de plans d'ensemble par des vues aériennes survolant Manhattan au son de la mélodie Fly Me To the Moon chantée en off par Frank Sinatra. Les hauts immeubles vitrés y sont embellis par la lumière dorée dénotant l'aurore, laquelle connote conjointement le luxe d'apparence d'une ville gouvernée par le pouvoir de l'argent ${ }^{15}$. A la faveur d'un fondu enchaîné, la deuxième partie de la séquence abandonne ce filtre d'enluminure et ces spectaculaires vues surplombantes pour représenter désormais la ville à l'échelle humaine, depuis les rames de métro et les rues sales et bondées où se côtoient les sans domicile fixe et les employés new yorkais irrités en route vers leur travail. La chanson désuète de Sinatra, en harmonie avec l'aspect aérien et enchanté des premiers plans, fidèle à un modèle cinématographique classique ${ }^{16}$, est soudain rendue "anempathique ${ }^{17}$ par la vision plus sinistre et réaliste de la rue. Avec cette opposition dialectique, Stone cherche à marquer le début du film de son empreinte ironique et insolente en opposant l'apparence mythique de la ville, telle que glorifiée notamment par le cinéma classique, et la réalité terne cachée derrière la surface apocryphe ${ }^{18}$.

Comment se positionne l'ouverture de World Trade Center par rapport à ces deux précédents filmiques? Il nous apparaît un point commun qui, s'il est peut-être tourné vers des enjeux dramatiques et emprunte des voies formelles chaque fois différentes, relie esthétiquement entre elles ces trois séquences : par un jeu d'oppositions, en creux, elles occasionnent d'entrée une déviation perceptive en orientant notre regard vers une réalité filmique sous-jacente dont la figure de New York se fait l'antichambre. Stone donne en premier lieu dans chacun de ces incipits une identité spécifique et différente de la ville en soustrayant du visible ce qui pourrait dénaturer le tableau descriptif. Ainsi, Wall Street réduit d'abord New York à la splendeur de ses immeubles d'affaires, et World Trade Center à la quiétude des quartiers résidentiels de la middle class.

En sautant du noir et blanc de l'ici à la couleur de l'ailleurs (Last Year in Vietnam), en coupant des plans d'ensemble dorés du fantasme urbain aux plans moyens ternis de la réalité de la rue (Wall Street), en entaillant, de façon plus ténue mais distincte, la sérénité chromatique par le signe de la menace (World Trade Center), Oliver Stone mesure l'écart entre les stéréotypes de la représentation urbaine et ce qu'ils refoulent. Cet écart est le fait d'un décentrement spatial et figuratif correspondant à l'idée de l'image-perception définie par Gilles Deleuze :

Le cinéma n'a nullement pour modèle la perception naturelle subjective [...], il parcourt le chemin dans les deux sens. [...] Nous allons de la perception totale objective qui se confond avec la chose à une perception subjective qui s'en distingue par simple élimination et soustraction (Deleuze, 94).

Dans World Trade Center, Oliver Stone trouble donc la représentation objective de l'aurore urbaine en focalisant momentanément notre regard, à plusieurs reprises et avec de plus en plus d'insistance, sur les tours du World Trade Center dressées en profondeur de champ. De fait, l'image du paysage a priori ordonné ne se donne plus à voir dans son objectivité première et actuelle: elle est affectée d'un facteur sensible, nous en sentons la fragilité, d'un facteur réflexif, nous soupçonnons qu'il s'agit d'une 
reproduction virtuelle, et d'un facteur temporel, nous anticipons l'imminence du danger.

Quand vous changez de format, explique Oliver Stone, vous rendez les spectateurs conscients du fait qu'ils sont en train de regarder un film, vous les secouez, vous modifiez les points de vue. Vous les déstabilisez, les désorientez. [...] Où est la réalité ? De quel point de vue s'agit-il ? Chaque moment extérieur possède son propre corolaire interne (Cieutat et Ciment, 13).

Dès lors, il est possible de lire ces trois incipits sous le prisme du mensonge dévoilé de l'Amérique telle qu'Oliver Stone la met en scène: l'expression du refoulement $d u$ souvenir dans Last Year in Vietnam, de la négation de la réalité sociale dans Wall Street ou enfin de l'ignorance du danger dans World Trade Center. En somme, trois thèmes coïncidant avec les problématiques socio-historiques majeures de chacune des trois décennies où se situent l'action de ces films, le traumatisme du Vietnam dans les années soixante-dix, la recréation scénarisée d'une Amérique mythique dans les années quatre vingt reaganiennes ${ }^{19}$, la " fin de l'histoire $~^{20}$ au tournant du nouveau siècle. Dans World Trade Center, le mode d'exposition progressif des tours jumelles numérisées dans le décor, en déliaison avec la figure humaine que seul le mouvement de caméra pousse à côtoyer, ne résorbe-t-elle pas à nos yeux de spectateurs la quiétude du monde diégétique en signalant combien celle-ci n'est qu'illusoire et temporaire? Encore une fois, notre propre perception subjective anticipe l'événement prochain et demeure sensible à une temporalité narrative quand bien même l'image ne nous fournit que des informations descriptives. Il est en effet notable que l'annonce de la dérive dramatique des événements n'est pas prise en charge par un quelconque effet de style ${ }^{21}$ ou par une action motrice qui gonflerait soudain la matière filmique d'une tension nouvelle, d'un suspense sans doute inconvenant étant donné l'issue inéluctable. Cet incipit introduit l'idée du danger latent sans avoir à s'y confronter déjà directement. Oliver Stone cherche à inventer un paysage en attente de son propre anéantissement ${ }^{22}$. Cette idée est dès lors inoculée en silence dans l'esprit d'un spectateur conscient, ou pas, de ce qui lui est raconté sous l'image.

\section{Dans l'ombre du spectacle}

Envisageons maintenant la rencontre entre les images filmiques et les images médiatiques préexistantes du désastre. L'impact du Boeing 767 American Airlines 175 contre la tour sud du World Trade Center étant devenu l'une des icones emblématiques des attentats du 11/9, comment Stone a-t-il résolu le nœud esthétique de sa transposition à l'écran? L'adaptation présente ici plusieurs difficultés. Après les séquences de préambule et de présentation liminaire du lieu de l'action, des personnages et de leur fonction narrative, la fiction va désormais rattraper l'Histoire. La séquence assurant cette fonction présente donc une double épreuve, à la fois pour le spectateur et pour le cinéaste.

D'abord, l'instant de quiétude fictionnelle décrit dans les premières minutes du film bascule inexorablement dans le chaos du vécu alors que le récit se confond avec le souvenir traumatique. L'appendice contemplatif et narratif précédant l'instant clé de l'attentat a peut-être pour objet de contrôler cette appréhension spectatorielle. Celle-ci s'est en effet manifestée plusieurs mois avant la sortie du film, dès la diffusion de ses premières bande-annonces ${ }^{23}$. Mon regard parviendra-t-il à soutenir une fois de plus les 
images meurtrières du $11 / 9$, à les voir revivre par le truchement biaisé du cinéma ? Serais-je capable de surmonter la douleur de la remémoration? La reconstitution n'estelle pas à même de trahir la captation télévisée de l'événement à laquelle j'ai assisté et par là même la dimension empirique de mon souvenir scopique? Bref, cette séquence est à même d'entraîner le spectateur dans l'épreuve du voir.

Ensuite, le réalisateur doit lui-même surmonter une épreuve en faisant se rencontrer le réel et la fiction. Cet épisode emblématique étant représenté au cinéma pour la première fois, l'image filmique conçue pour l'occasion scelle cette transposition d'un régime iconique à l'autre et sera peut-être appelée à devenir pérenne. De plus, l'engagement de cinéaste d'oliver Stone, comme il lui a été demandé avec insistance par la Paramount, lui impose de respecter en premier lieu la mémoire collective. La démarche esthétique délivrée par le cinéaste s'annonce donc comme le fruit d'une épreuve éthique.

Will Jimeno effectue une ronde de patrouille matinale dans une rue du centre de Manhattan, à la sortie de la très fréquentée Penn Station. Son attention est attirée par trois actions simultanées et anodines. Un groupe de sans domicile fixe encombre un lieu public, un bonimenteur importune une passante et un groupe de touristes demande son chemin. Rien qui ne relève pas du quotidien de la profession de Jimeno en même temps que d'une vieille convention cinématographique ${ }^{24}$. Ce court effet de dispersion dramatique fait office de diversion jusqu'à ce qu'une brusque éclipse de soleil, accompagnée d'un sourd vrombissement étranger au tumulte urbain, impose soudain le silence et invite tous les regards à se tourner vers le ciel, hors-champ. Le contrechamp ne révèle pas l'objet de l'ombrage et du surgissement sonore. Que nous montre alors l'image en lieu et place de l'avion se projetant vers sa cible? Son ombre fugace se reflétant sur la façade jaune d'une salle de cinéma.

A l'égard d'un événement dramatique indubitablement attendu, avec impatience ou appréhension, par les spectateurs, Oliver Stone manifeste ostensiblement son refus du spectaculaire. Le cinéaste se place en retrait par rapport aux codes hollywoodiens qui, sous la pression d'un ancrage générique plus marqué, auraient exigé le concours d'une pyrotechnie tonitruante. Stone propose une économie figurative métaphorique: le contexte fictionnel de l'adaptation cinématographique ne peut nous donner à voir que l'ombre de la réalité. Le recours quasi littéral au trompe-l'œil platonicien retourne comme un gant l'image spectaculaire et en garde l'inscription spéculaire, rejetant l'illusoire vérité que la représentation pourrait revendiquer. La façade de la salle de cinéma ${ }^{25}$ sur laquelle est projetée l'ombre portée ne satisfait pas aux attentes scopiques du spectateur, elle expose sur un mur-écran l'image même de cette attente à jamais irrésolvable. L'évocation visuelle et sonore implicite de l'impact doit suffire à faire émerger en nous les images que ce plan occulte. Pour le dire avec Stéphanie Katz, « en investissant les béances de la figure, le spectateur s'implique dans un processus d'invention de l'infigurable " (Katz, 30). Si cette ombre possède un référent dans le monde diégétique et dans l'imaginaire spectatoriel, l'avion survolant la ville à basse altitude, elle n'a pas de référent dans la réalité filmé, elle n'est que pur trucage optique. Oliver Stone ne se départit donc pas pour autant de toute logique hollywoodienne et cherche à restituer par l'ellipse l'art de la synthèse cinématographique: il fait de l'ombre l'emblème d'une position éthique, il convertit l'irreprésentable en icône figurative de l'immontré. 


\section{Le défi de l'image régnante}

29 La troisième séquence emblématique de l'approche esthétique du 11/9 par Oliver Stone se situe quelque instants après la collision, éludée à l'écran, du vol American Airlines 11 contre la tour Nord. Cette fois-ci, les premières images de la catastrophe vont apparaître. L'action se situe dans une salle commune du commissariat où Jimeno, McLoughlin et les autres membres de la brigade ont été réunis en urgence sans savoir encore de quoi il retourne. Quatre plans précèdent le dévoilement des premières vues de l'attentat. Assis face caméra, deux policiers en uniformes cadrés en plans rapprochés murmurent une conversation inaudible, le regard tendu et concentré vers le horschamp devant eux. De cet espace encore invisible s'élève beaucoup plus nettement la voix de Carol Lin, la présentatrice télévisée de la chaîne CNN décrivant en direct la scène du drame. Au deuxième plan, deux nouveaux policiers échangent également des regards incrédules et des mots à voix basse au devant de la caméra, avant que celle-ci panote vivement vers la droite pour accueillir et dramatiser l'entrée en scène du capitaine de la brigade, distinct de ses hommes par sa chemise blanche immaculée, lequel vient se poster mains sur les hanches au centre du cadre pour proférer à voix haute un juron en réaction aux images diffusées devant lui et que nous voyons toujours pas. Le plan suivant ne nous offre pas de contrechamp vers l'écran de télévision, il nous place derrière Jimeno et son coéquipier suivis en steadycam alors qu'ils approchent de la pièce silencieuse. Quand ceux-ci tournent au fond du couloir pour passer la porte, nous nous attendons à découvrir en même temps qu'eux la télévision qui diffuse les images, mais un changement de plan retarde encore un peu plus cette vision. Nous reprenons les personnages de face découvrant bouche-bée la catastrophe. Ce n'est qu'au cinquième plan de cette séquence que le contrechamp nous est dévoilé, par dessus l'épaule des policiers emplissant la pièce, après que l'un d'entre eux soit sorti du champ, ultime effet de rideau masquant le spectacle. La télévision est allumée sur CNN et présente l'un des fameux plans filmés par hélicoptère du World Trade Center percé d'une embrasure béante.

30 Alors que la reconstitution cinématographique de l'impact nous a été refusée quelques séquences auparavant, pourquoi retarder désormais à ce point la vision d'images d'archives qui nous sont $a$ priori familières ? Pourquoi ce suspense soudain à l'égard d'un spectacle médiatique dont nous n'ignorons presque rien? Le dispositif filmique articule deux ingrédients propres à la dramatisation, un écran second et un regard médiateur. Christian Metz a proposé l'expression «écran second » pour nommer les types de dispositifs par lesquels le cadrage filmique est redoublé dans l'image par un encadrement matériel du visible (fenêtre, embrasure de porte, rideaux, etc.) qui restreint notre perception et met en valeur l'objet filmé sous forme de tableau.

Le foyer du film, en somme, redouble la médiation scopique [...] Le cadre intérieur second, a pour effet de mettre en évidence le premier, c'est-à-dire le lieu de l'énonciation dont il est, parmi d'autres, une "marque» fréquente et reconnaissable (Metz, 73)

31 S'il est un cadre second à considérer ici dans cette logique du surcadrage, c'est peutêtre moins celui de l'appareil de télévision branché sur la retransmission de l'attentat que l'encadrement physique de cet objet par les silhouettes des spectateurs diégétiques dressés tout autour. Le cadre filmique n'épouse pas le cadre télévisé, la source énonciative est au contraire toujours ancrée au sein de cette communauté de regards que la caméra n'éclipse jamais. «Le jeu des écrans seconds pourrait être compté au 
nombre de multiples procédés (...) permettant de mettre en évidence le » dispositif « du cinéma » (Metz 93-94). Ainsi Oliver Stone n'a-t-il pas pour ambition de recréer avec les moyens du cinéma les conditions du spectaculaire à l'égard d'un événement ayant déjà prouvé son pouvoir de sidération. Ce que nous semble chercher ici à mettre en scène son geste cinématographique, c'est justement l'instant épiphanique et mobile de l'événement en tant qu'il est d'abord un événement scopique. L'enchaînement des quatre plans précédant les images télévisées de l'attentat ne retarde pas tant ce contrechamp pour accroître la tension de notre découverte, il veille plutôt à aménager petit à petit dans le cadre un empilement croissant de corps et de regards, condensation affective exacerbant la stupeur partagée avec laquelle ces images sont intradiégétiquement reçues. Autrement dit, l'objet premier du spectacle filmique n'est pas le regardé sidérant mais le regardant sidéré.

Un principe de construction communautaire est à l'œuvre derrière ces images d'archives. Celles-ci convoquent le souvenir spectatoriel dans la fiction afin de permettre à la fois d'authentifier le récit et de favoriser l'identification. Nous sommes en présence d'une archive prélevée sur le réel, image-trace, mais également d'une vue filtrée à travers le regard des personnages qui la découvrent, image-médiatisée. "L'effarement contemplatif (des figurants mais aussi du spectateur) constitue la réponse à l'événement " analyse André Gardies lorsqu'il étudie le spectaculaire au cinéma (Gardies 125). Mais lorsque cette réaction vaut quasiment pour seul spectacle, comme c'est le cas dans cette séquence où les images de l'attentat restent fugacement dévoilées sur un écran de télévision tandis que les regards effarés occupent bien plus notre attention, le dispositif filmique met en avant le caractère phénoménal de la situation narrative sans chercher à reproduire sur nous la même stupeur que celle lue sur les visages des personnages.

Les attentats du 11/9 doivent donc être considérés à ce stade comme un événement diégétique et non comme un événement filmique ${ }^{26}$. En effet, il sont médiatisés par un outil optique (le poste de télévision) et racontés par un narrateur (la voix de la présentatrice) tous deux intradiégétiques et sur lesquels le spectateur a, supposément, plus de savoir. En revanche, la situation vue et narrée ne surgit pas à nos propres yeux comme une épreuve figurative puisque le film omet sciemment de la représenter. Dès lors, les images-traces, ou images-documents, des attentats se trouvent enchâssées par l'espace et la temporalité du monde diégétique. Le récit rejoint donc l'Histoire par la simultanéité dramatique entre l'action englobante et l'image-document actualisée par la diégèse. La télévision, qui agit ici comme outil optique de mise en abyme, n'attesteelle pas de l'appartenance indéniable de ces images au passé ?

\section{Déchirer l'écrin du visible}

Cependant qu'il a favorisé l'esquive figurative pour inaugurer le traitement formel de l'adaptation, Oliver Stone s'enjoindra-t-il par la suite à affronter ouvertement l'épreuve de la reconstitution iconique? La direction prise par le récit peut difficilement l'encourager à persévérer dans une logique du moindre effet. Tôt ou tard, il faudra montrer. La dernière séquence que nous voudrions maintenant étudier met en scène l'entrée des secouristes dans la tour, juste avant son effondrement. Oliver Stone pourrait être incité par son sujet et par les possibilités offertes par la reconstitution pour inaugurer avec World Trade Center une représentation alternative du 11/9. Le 
cinéaste étant semble-t-il réfractaire à la possibilité d'usurper aux images médiatiques leur pouvoir spectaculaire, il a au moins licence pour contrarier leur caractère occultant. Ainsi est-il en mesure de montrer ce qui jusque-là nous avait échappé, en situant l'action de cette séquence en un lieu d'où peu d'images nous sont parvenues, le parvis du World Trade Center peu avant l'effondrement de la première tour. Nous avons vu le rôle joué par les archives télévisées, voyons désormais si la représentation frontale du 11/9, sans le recours à ce type de béquilles figuratives, reste encore en partie tributaire du faisceau de représentation à l'intérieur duquel la visibilité du 11/9 s'est façonnée.

Les procédés d'évitement et d'annexion du visible, qui gouvernaient les deux précédentes séquences étudiées, laissent désormais place au démarquage. Au terme du premier quart d'heure du film, l'équipe de secouristes parvient enfin au pied des tours du World Trade Center. Depuis l'arrivée des personnages sur les lieux jusqu'à leur entrée dans la tour sud, la séquence comporte soixante plans. Oliver Stone semble envisager la mise en scène en décalage avec la situation chaotique et frénétique de la catastrophe. La majorité des plans sont de longs plans moyens ou rapprochés centrés exclusivement sur les secouristes, attendant leurs ordres dans un premier temps, s'élançant vers le World Trade Center dans un second temps. La caméra retranscrit alors leur stupeur interdite en balayant ou en fixant dans le cadre, en contre-plongée, leurs visages hébétés levés vers les tours en hors-champ. Neuf plans tournés vers le contrechamp attirent alors plus particulièrement notre attention en cela qu'ils nous donnent à voir l'immeuble en feu et les victimes blessées au sol, soit tout ce que la représentation nous avait jusque-là refusé. Or, pour décrire la scène de l'attentat et nous en rendre une nouvelle visibilité filmique, la mise en scène fait appel à des tropes formels à usage unique, jusque-là inusités dans le film et qu'Oliver Stone ne réemploiera pas plus tard.

En effet, à la faveur de ces plans exceptionnels, les premiers rescapés évacuant les tours sont filmés à l'épaule et hâtivement recadrés par zoom, par une caméra imitant soudain la prise de vue sur le vif d'un reportage ou d'un documentaire. Si l'agitation du cadreur n'est pas justifiée narrativement, le mouvement désorienté de la caméra ne renvoyant ni à une vision subjective ni à un appareil intradiégétique, elle n'est pas non plus justifiée formellement puisque les plans intercalaires restent cadrés avec stabilité et retenue. Nous pouvons alors parler d'un faux raccord esthétique, relevant en fait d'un procédé d'imitation. Les plans filmés à l'épaule, bien que composés au tournage, réfèrent ici aux images saisies à l'arrachée par les caméras de presse ou par les cameramen amateurs le matin du 11/9 et sempiternellement rediffusées sur les chaînes de télévision pour illustrer la catastrophe. Cette citation formelle institue de fait la représentation médiatique en une visibilité normative de l'événement que le cinéaste prend le parti d'imiter plutôt que de lui substituer de nouvelles modalités du voir. En bref, Stone use a priori arbitrairement d'un stéréotype visuel associé au 11/9. Nous dirons qu'il procède à une imitation canonique. Est-ce pour faciliter pour autant notre réception des images filmiques? La suite de la séquence peut permettre d'en douter.

En effet, plus loin, en contrechamp du regard levé de Jimeno, Stone interpole un plan zoomé tourné à l'épaule dans lequel nous suivons d'un mouvement panoramique descendant la chute de l'une des victimes désespérées le long de la paroi de la tour. De tels plans ont été réellement filmés et diffusés en direct par les médias le 11/9, mais à quel titre sont-ils sollicités par Oliver Stone ? Clément Chéroux rappelle que « quantité 
de photographes, des professionnels comme des amateurs, fixèrent l'image de ces » jumpers « comme les appelle la presse américaine » (Chéroux, 38), mais que ces photographies n'avaient eu que rarement droit de cité dans la presse écrite ${ }^{27}$. Une photographie de Richard Drew intitulée Falling Man est restée célèbre pour avoir capturé les derniers instants d'un homme vêtu de blanc chutant tête la première à la verticale de la tour. Cette image fut publiée dès le 12 septembre dans plusieurs colonnes de journaux ${ }^{28}$. Mais, hormis ce cas particulier, étant donné que «les photographies des blessés, des défenestrés et des fragments humains montrent directement la souffrance des victimes, l'imminence de leur mort ou la dislocation de leur intégrité corporelle » (Chéroux, 39), elles furent majoritairement bannies par la presse pour leur pénibilité visuelle. Ainsi, il apparaît, selon Chéroux, que «ce filtrage des photographies les plus violentes a bel et bien participé à la raréfaction des représentations de l'événement et partant à l'uniformisation de son traitement médiatique » (Chéroux, 44). En amont de toute adaptation, l'identité visuelle du 11/9 serait donc déjà bâtie sur une vision sciemment parcellaire de la catastrophe, encouragée par l'autocensure, standardisée autour d'une logique de l'édulcoration et donc bornée par les limites arbitraires de l'immontrable. Au même titre que Clément Chéroux, Gerry Canavan remarque que :

Footage of the jumpers, despite their striking visual qualities, have been rare or non-existent in the major cultural reproductions of September 11. [...] The video footage, seen on live TV at the time, is generally not played during annual September 11 retrospectives. [...] This silent self-censorship is all the more startling given the undeniable vividness of the memory of the jumpers for those who saw the coverage live on September 11, 2001 - the elimination of the jumpers has become something like mandatory cultural amnesia, a refusal on the part of the spectaclemakers to acknowledge a powerful image they now wish they had never showed us (Canavan, 124).

Cette hypothèse de « l'amnésie culturelle » peut être nuancée en rappelant que, si l'idée de violence et de dégradation corporelle induite par l'image des jumpers a été rapidement soustraite de la représentation médiatique du 11/9, la culture populaire n'a pas pour autant éradiqué toute référence à ce motif visuel. Par exemple, dans le courtmétrage de Alejandro Gonzales Iñaritu réalisé pour le film collectif 11'09'01 (2002), des images subliminales des jumpers déchirent progressivement le noir qui recouvre la totalité de l'écran. Dans le roman graphique et semi-autobiographique A l'Ombre des Tours Mortes (In the Shadow of no Towers, Art Spiegelman, 2004), l'auteur dessine dans la bande verticale d'une marge son propre avatar graphique tournoyant devant une tour embrasée, référence à ces corps suicidaires dont le narrateur se dit obsédé pour ne les avoir pas vus (Spielgelman 6). L'artiste Eric Fischl a réalisé sur le sujet une œuvre qui fit polémique, une sculpture de bronze représentant un corps de femme nu, plié et renversé, bras et jambes au-dessus de la tête, figé en position de chute (Tumbling $W_{o m a n}{ }^{29}$ 2002). La photographie de Richard Drew donne également son titre au roman de Don De Lillo, L'Homme qui Tombe (De Lillo 2008) situé dans le contexte immédiat de l'après-catastrophe à New York. Place a donc été trouvée dans la culture de l'après-11/9 à cette image du corps en chute libre, précipité vers sa mort imminente. A la lumière des exemples cités, nous pouvons néanmoins noter que le motif « interdit » du jumper sur lequel se reporte le souvenir du 11/9 ne contribue pas à l'exigence additionnelle d'une visibilité exhaustive de l'événement, il nous semble plutôt employé pour dénoncer l'incomplétude de la représentation canonique qui d'ordinaire le récuse. 

poussé ces désespérés vers un tel geste ultime. Ce sont en revanche les plans fugaces ou encore les photographies ayant saisi et figé à la volée l'instant transitoire de leur chute qui sont cités. Ces images présentent un caractère tout à la fois excédentaire et lacunaire. Excédentaire car, simultanément, elles exhibent la mort à l'œuvre et rappellent l'insoutenable impuissance de ma position de spectateur, elles connotent le désespoir et le renoncement tragique de ces victimes du drame, elles exacerbent la froide suprématie du désastre face auquel l'existence humaine affiche une si dérisoire précarité. Mais d'un autre côté, ces images se révèlent lacunaires car elles ne fixent que des silhouettes à la lisière de la désincarnation. Le corps en chute libre résiste à la disparition en une figure-frontière, pas tout à fait dépolie de son humanité mais plus tout à fait unique. Ainsi, les individus se devinent encore et se dissipent déjà dans la forme sérielle et itérative de ces "virgules noires » tombant du ciel, ainsi que les décrivait le témoin oculaire James Whitney ${ }^{30}$. Le motif est encore lacunaire pour nous laisser anticiper sans équivoque le devenir de ces corps dont nous ne verrons jamais les lambeaux joncher le sol. En somme la figuration du jumper soumet l'image à une tension dialectique entre l'excédent et la carence de visible qui l'empêche de trouver place dans les représentations médiatiques normées de l'événement. Alors que les vues spectaculaires de l'explosion et de l'effondrement des tours font de l'architecture éventrée le signe métonymique et déréalisé de la mort à l'œuvre, pensée comme rapide, multiple et simultanée, l'individuation des corps plongeant dans le vide nous ramène à sa réalité physique en lui accordant la densité d'une temporalisation et d'une incarnation, et nous rappelle l'aporie figurative qui affecte la visibilité canonique de l'attentat. Au sujet de la métaphore des "virgules noires ", Thierry Jousse affirme que cette expression imagée "porte en elle une dimension inoubliable, une dimension immémoriale que la télévision et son ressassement ne connaissent pas. Elle seule peut déjouer la dictature du temps réel dont nous sommes tous intoxiqués. Elle seule donne enfin la dimension figurative qui nous manquait pour concevoir que l'impossible a été possible» (Jousse, 11). En restituant le temps d'un rapide plan de World Trade Center, l'image raréfiée d'un jumper, Oliver Stone procède à ce que nous nommons une citation clandestine, qui repositionne le récit dans le temps présent et ineffable de l'événement scopique lors duquel les corps suicidaires n'étaient pas encore cachés et ma vision pas encore délibérément bridée. Ne dénonce-t-il pas par la même occasion l'autocensure médiatique qui a sévi ultérieurement?

Gerry Canavan critique pourtant la reconstitution filmique de World Trade Center comme un nouvel exemple d'autocensure qui contribuerait à édulcorer la violence de l'événement (Canavan,128). Pour Canavan en effet, les secouristes aux pieds des tours, dont nous suivons les déboires, devraient être en position de voir au sol les cadavres disloqués des suicidés. Le refus de ces visions horrifiques, qui seraient pourtant justifiées diégétiquement, tendrait à déréaliser encore une fois la représentation. Certes, Oliver Stone situe l'action de cette séquence dans un lieu d'où peu d'images nous sont parvenues, et cette position pourrait l'autoriser à montrer ce qui jusque-là nous avait échappé. Pourtant, comme nous l'avons vu plus haut, le cinéaste n'adopte pas le point de vue des témoins oculaires: il substitue à l'image de leur vision, en contrechamp à leur regard, l'imitation canonique d'une captation filmique en direct. Le motif clandestin du jumper pourrait plaider en faveur d'un cinéaste semble-t-il désireux de ramener dans le champ de visibilité ce qui en avait été chassé. Pourtant, Canavan ne s'estime pas convaincu par la présence fugace de ce plan qui se substitue à toute vision 
de cadavre. Or, il ne nous semble pas que l'ambition esthétique du cinéaste ait été de combler la carence des images médiatiques par des suppléments figuratifs et fictionnels. Plutôt que de nous donner un plein accès à la visibilité de la catastrophe, Stone limite sciemment notre regard à des tropes audiovisuels déjà consommés, comme le montre la suite de la séquence.

41 Ainsi, lorsque les secouristes s'engagent à pied vers le hall de la tour, ils traversent un champ figuratif uniquement composé d'ingrédients visuels issus de représentations médiatiques ou documentaires préexistantes. Un homme d'affaire portant un attachécase et un costume sombre accoste l'équipe de secouristes pour leur communiquer avec inquiétude quelques informations. Son visage et ses cheveux grisonnants sont entièrement noircis par la suie. Quelques instants plus loin, lorsque John McLoughlin s'élance dans la rue à la tête de sa petite escouade, Oliver Stone alterne en champ/ contrechamp le regard du héros et ce qu'il perçoit en vue a priori subjective. La caméra fixe alors son attention sur un objet incongru que le personnage croise sur sa route: une roue de l'un des avions, déchirée, fumante, appuyée au coin de la rue contre un échafaudage. Immédiatement après, dans le même plan, la caméra est attirée par la présence d'une jeune femme en jupe et chemisier rouge, le visage couvert de sang, qui court en pleurant avec hystérie sous une pluie de débris de béton. Plus loin, une fois rentrés dans le hall de la tour, les secouristes sont à plusieurs reprises interrompus dans leur dialogue par de lourds claquements secs en hors-champ, dont l'origine acoustique ne nous est pas révélée.

Souillé par les scories de l'explosion, l'homme d'affaire élégant ne porte plus sous les cendres et la suie que le spectre de son identité physique et sociale. La roue de l'avion de ligne éclatée contre un trottoir, objet dissonant dans le décor urbain, se veut source d'étrangeté et d'inquiétude. L'apparition horrifique du premier corps ensanglanté est signe d'une violence organique, redoublée par les couleurs rouge du costume et des camions de pompier bouchant le second plan, qui a pour vocation de produire un bref choc esthétique. Enfin, les claquements permanents entourant les personnages connotent l'idée d'un monde en train de s'écrouler sans résistance autour d'eux. Oliver Stone ne montre quasiment rien de plus du désastre humain que ces quelques figuressymptômes qui absorbent et résument à elles seules la démesure de la catastrophe et l'état d'annihilation du commun dans lequel la réalité filmique est plongée. Il est alors essentiel de constater qu'aucune de ces figures n'a été inventée pour le film, mais que toutes sont des transpositions à l'écran de critères déjà présentés dans la presse ou les documentaires réalisés sur l'événement. Ainsi, l'homme d'affaire à la mallette, en plus d'évoquer les silhouettes génériques des employés évacués du World Trade Center, semble donner vie à la statue de bronze intitulée Double Check située à Liberty Plaza Park dans le Lower Manhattan, à proximité des tours jumelles. Créée par J. Seward Johnson Jr en 1982, cette œuvre grandeur nature représente un homme en costume, assis sur un banc, vérifiant le contenu de sa mallette avant de se rendre au travail. Endommagée et couverte de débris après les attentats, la statue fut, dit-on, confondue par certains sauveteurs avec un véritable blessé (Pollak). Par la suite, la presse s'en fit amplement l'écho, elle devint un emblème du $11 / 9$ et retrouva sa place initiale après sa restauration par l'artiste. De même, la vision de la roue de l'appareil que McLoughlin aperçoit cite une véritable photographie prise par Charles Marsh le 11 septembre $^{31}$, employée notamment par le NIST's World Trade Center Investigation ${ }^{32}$ pour déterminer précisément la direction de l'appareil au moment de l'impact. La femme en rouge, elle aussi, apparaitt sur de véritables clichés, notamment celui capturé par la reporter Ruth 
Fremsen et publié en Une du New York Times, le 12 septembre 2001 (Fremsen, A1) ${ }^{33}$. Quant au bruit inconnu provoquant un malaise muet chez les personnages, son origine a été fixée et élucidée par le documentaire réalisé sur place le 11 septembre 2001 par les frères Jules et Gédéon Naudet $(11 / 9,2001)$ : il s'agit du claquement que font entendre les corps des suicidés heurtant continument le sol, motif sonore auquel oliver Stone fait référence sans l'expliquer.

Stone s'emploie donc à reconstituer le $11 / 9$ en restituant l'identité audiovisuelle de l'événement, plutôt que par l'invention figurative et sonore. Faut-il admettre en fin de compte que le cinéma prononce là son échec à s'emparer pleinement de la représentation? L'adaptation est-elle donc subordonnée à l'autorité médiatique et documentaire jusqu'au refus de toute extension de l'imaginaire fictionnel? Les exemples cités concourent effectivement à constater qu'oliver Stone ne s'est engagé à prêter aux figures empruntées à l'imagerie médiatique ni antécédents ni devenir. Il se limite dans cette introduction à modéliser et à animer les photographies de presse et leurs figurants sans jamais les faire déborder dans la fiction, sans inventer une histoire à leur présence. Malgré les possibilités narratives proposées par l'événement le cinéaste se consacre à décrire la mésaventure dramatique de deux secouristes isolées dans le chaos. Dès lors, si les bornes du récit empêchent sciemment et logiquement ce dernier d'explorer la diégèse en profondeur, il pourrait paraître surprenant que cette limitation narrative s'accouple à une représentation si canonique de l'événement. Que John McLoughlin, d'un seul balayement de regard, puisse apercevoir deux figures issues de photographies de presse cardinales joue contre la vraisemblance. Dès lors, il est possible d'évoquer à l'égard de ce principe de reproduction figurative une greffe d'authenticité.

Ainsi, le langage cinématographique provoque l'illusion d'un contact entre la fiction et la réalité, qui ne signe pourtant la fidélité de l'adaptation qu'à l'iconographie consacrée de l'événement. Or, à notre sens, en ne construisant la reconstitution qu'à partir d'emprunts documentaires, Oliver Stone n'a pas vocation à traduire la vérité du vécu mais à désigner l'impossible rencontre de la fiction hollywoodienne et de la tragédie réelle. La multiplication des champs contrechamps entre les regards ébahis des personnages et les images du drame reconstituées marquent la déliaison entre deux hétérogénéités, le corps et son environnement, qui ne sauraient se rejoindre dans l'action motrice. John McLoughlin traverse l'espace scénographique sans que jamais sa nature héroïque ne se révèle au contact de l'événement. Si les contrechamps sur les tours en flammes ou sur les victimes en sang font office de greffe d'authenticité, c'est peut-être pour mieux marquer le rejet du présent narratif et du passé historique, figé par l'image médiatique, sur lequel le premier ne pourrait avoir d'influence dans le récit que mensongère. L'élan corporel du héros n'est jamais en mesure de créer une impulsion dynamique sur son environnement, ou il risquerait d'engager dans l'esprit du spectateur l'idée apocryphe d'une résolution positive de son action. Stone emploie donc un montage non pas basé sur l'interaction des parties mais sur leur indifférence physique. De fait, le cinéaste revendique la vacuité de l'adaptation, laquelle ne peut selon lui revisiter l'Histoire qu'en se confrontant à ses images. Stone semble vouloir signifier que face à cet événement tragique, les héros impuissants de World Trade Center en témoignent, il n'existe pas d'autre position que celle de spectateur. Ne pas profiter de la reconstitution filmique pour montrer abondamment ce que les médias ont censuré, pour creuser les promesses fictionnelles de la catastrophe ou pour investir son champ figuratif, telle est la réponse éthique d'oliver Stone aux problématiques de 
l'adaptation. Construire la reconstitution du 11/9 comme un corps narratif englobant reviendrait à trahir son essence événementielle en instaurant un savoir qui était originairement absent. De cette démarche découle sur le plan esthétique l'imitation des images primitives de l'événement et la recherche de l'épure formelle où seules certaines icones absorbent et résument à elles seules la démesure de la catastrophe (l'ombre sur le gratte-ciel), son retentissement (les corps agglutinés devant l'écran de télévision), sa violence (le visage maculé de sang), son aporie (le désespéré se jetant dans le vide).

\section{Conclusion}

Impossible pour Oliver Stone d'altérer l'identité iconique du 11/9. Le cinéaste se fournit assez scrupuleusement dans ce que nous pourrions nommer le «catalogue du visible ", produit notamment par la presse dans les quelques jours suivant les attentats et auprès duquel la mémoire collective se réfère encore en permanence. La paralysie de la représentation, incarnée dans les médias par l'autocensure, n'a pas été levée par Oliver Stone. Celui-ci s'est au contraire refusé à représenter autre chose que ce que nous avions déjà vu. De même que l'aspect humain et moral de l'histoire fut diversement apprécié aux Etats Unis ${ }^{34}$, la restriction intentionnelle du champ de visibilité généra des critiques. Stephen Prince remarque notamment que «sans s'affranchir des points de vue des personnages principaux, sans adopter une vue plus large, en demeurant à l'intérieur de son cadre microcosmique, [Stone] ne peut créer aucun dialogue entre les différentes perspectives soulevées par les événements que le film dépeint $»^{35}$ (Prince, 2009, 104). Par ailleurs, comme nous avons essayé de le montrer, les vingt premières minutes du film en partie étudiées dans cet article s'attachent à nous projeter dans une galerie visuelle et sonore au sein de laquelle les personnages, malgré leur position héroïque de secouristes, sont constamment des témoins, jamais des actants. Le travail de reconstitution proposé dans World Trade Center vise à éviter toute dramaturgie, il n'occulte jamais l'idée essentielle que le spectateur n'est pas ignorant de l'issue de la tragédie et ne cherche jamais à créer l'illusion du doute par une appropriation dramatique de son déroulement. Sans doute peut-on saisir en quoi cette consécration de l'événement scopique matriciel a pu être perçue comme un désaveu du pouvoir cinématographique, apparemment incapable de présentifier le drame, préférant le tenir toujours à distance par un regard médiateur. En France, Antoine de Baecque reproche quant à lui au film d'être «en permanence dans une distance reconstitutive qui transforme chaque regard en clin d'œil, chaque uniforme en fétiche morbide » (De Baecque, 430). La fidélité de l'imitation figurative, seulement réservée aux représentations primitives de l'attentat comme en témoigne l'évocation des suicidaires, nous paraît pourtant être plus qu'un clin d'œil fétichiste, elle en montre plutôt l'inaltérable qualité iconique contre laquelle le cinéma ne peut qu'achopper. Plutôt que d'envisager la reconstitution mise en place par Oliver Stone comme une collecte soignée de détails, il nous a semblé préférable de montrer comment World Trade Center cherche avec difficulté son identité esthétique au cœur d'un faisceau de représentations dans lequel il lui convient de définir sa juste position. Au centre de la menace qui plane sans se manifester sur le paysage, dans l'ombre du cataclysme, derrière la lucarne télévisée qui permet d'apercevoir par bribes les conséquences du désastre, entre les images reconstituées du chaos, c'est le décentrement du regard qu'a donc choisi Oliver Stone pour figurer le 11/9. Sa nature véritable ne cesse donc jamais 
d'être pour l'image un événement périphérique. A l'instant de la chute des tours, Stone renforce encore cette perspective qu'il tient jusqu'à la fin du film, en déplaçant le regard du spectateur dans le gouffre atrophié et obscur où gisent les corps anéantis, définitivement inaptes à l'action, piégés dans le seul espace scénique depuis lequel aucune vision n'est possible. Les vingt premières minutes de World Trade Center cristallisent de manière signifiante l'aporie de la représentation hollywoodienne $\mathrm{du} 11 / 9$, tendue entre la pulsion scopique et le refus d'en montrer davantage. Pour terminer, nous devons considérer que ce nœud esthétique, parce qu'il tend à entrelacer le visible et l'invisible de la catastrophe dans le seul paradigme éthique de l'immontrable, rejette tout le contexte causal socio-historique dans les abîmes de l'impensé. Oliver Stone place son film sous l'allégeance de la sidération initiale, qui paraît incarner dès lors l'essence même du 11/9. L'éthique du décentrement est donc également corolaire d'une politique de la décontextualisation. Evénement dans l'événement, la destruction du World Trade Center reste en effet l'épisode le plus surmédiatisé des attentats, un noyau iconique encore infrangible en périphérie duquel se construit ici le récit filmique, dans l'ombre du visible, dans les replis de l'image, mais aussi dans l'angle mort de l'Histoire.

\section{BIBLIOGRAPHIE}

Arquembourg, Jocelyne. «Le mythe de Pandore revisité » . In La terreur spectacle. Terrorisme et télévision. Ed. Daniel Dayan. Paris : De Boeck, «Collection Média Recherches » Paris : 2006. 79-85. Atkins, Stephen (Dir.). The 9/11 Encyclopedia, Second Edition. Santa-Barbara: ABC/CLIO, 2011. Baudrillard, Jean. Amérique. Paris : Editions Grasset et Fasquelle, Le Livre de Poche, 1986.

---. L'Esprit du terrorisme. Paris : Editions Galilée, 2002.

Berthomieu, Pierre. Hollywood moderne, le temps des voyants. Paris : Rouge Profond, 2010.

Bessalel, Jean et Gardies, André. 200 mots-clés de la théorie du cinéma. Paris : Cerf, 1992.

Borradori Giovanna, Derrida, Jacques et Habermas, Jürgen. Le « concept » du 11 septembre. Dialogues à New York (octobre-décembre 2001) avec Giovanna Borradori. Paris : Galilée, «La philosophie en effet », 2003.

Bragard, Véronique, Dony, Christophe et Rosenberg, Warren. Jefferson: McFarland \& Company, Inc., 2011. 118-133.

Canavan, Gerry. « Resignifying September 11 in World Trade Center and United 93 ». In Portraying 9/11. Essays on representations in comics, literature, film and theatre. Ed.

Cettl, Robert. Terrorism in American Cinema: An Analytical Filmography, 1960-2008. Jefferson :

McFarland \& Co Inc, 2009.

Chéroux, Clément. Diplopie. L'image photographique à l'ère des médias globalisés. Essai sur le 11 septembre 2001. Paris : Les Editions du Point du Jour, 2009.

Chion, Michel. Le son au cinéma. Paris : Cahiers du Cinéma, Essais », 1985. 
Cieutat, Michel et Ciment, Michel. « L'Amérique, terre de l'échec. Entretien avec Oliver Stone ». Positif, n 422, avril 1996. 10-14.

De Baecque, Antoine. L'Histoire-Caméra. Paris : Gallimard, « Bibliothèque illustrée des histoires », 2008.

Deleuze, Gilles. L'image-mouvement. Paris : Editions de Minuit, 1983.

De Lillo, Don, L'Homme qui Tombe, Paris : Babel, 2008 [2007].

Drew, Richard. « Falling Man ». New York Times, vol. CL, n 51874, 12 septembre 2001: A7.

Fremsen, Ruth. «Sans titre », New York Times, vol. CL, n 51874, 12 septembre 2001 : A1.

Fukuyama, Francis. La Fin de l'histoire et le Dernier Homme. Paris : Flammarion, « Champs », 1992.

Gardies, André. « Le moment spectaculaire dans la narration cinématographique ». In Le spectaculaire. Ed. Christine Hamon-Siréjols et André Gardies. Paris : Aléas Editeur, 1997. 119-128.

George, Pierre. « Les virgules noires ». Le Monde, n 17614, 13 septembre 2001.

Gervais, Bertrand. « Déjouer le spectacle de la violence. Représenter les événements du 11 septembre 2001 ». E-rea, revue électronique sur le monde anglophone, 9.1. 11 septembre 2011. Consulté le 14 septembre 2011.

<http://erea.revues.org/1944>.

Grossvogel, David I. Scenes in the city, Films vision of Manhattan before 9/11. New York : Peter Lang Publishing, 2003.

Jost, François. Le Temps d'un regard, du spectateur aux images. Québec, Paris : Nuits Blanches Editeur, Méridiens Klincksieck, 1998.

Jousse, Thierry. « New York, 11 septembre, l'envers du spectacle ». Cahiers du Cinéma, n 561 , octobre 2001. 10-11.

Katz, Stéphanie. L'écran, de l'icône au virtuel. La résistance de l'infigurable. Paris : L'Harmattan, 2004.

Lanzmann, Claude. «Claude Lanzmann : sur le courage. Entretien recueilli par Patrice Blouin, Franck Nouchi et Charles Tesson ". Cahiers du cinéma, n 561, octobre 2001. 46-57.

Lopez, Kathryn Jean. « Too soon ? United 93 provides a reality check ». 3 mai 2006. Consulté le 12 juin 2011.

<http://www.nationalreview.com/articles/217516/too-soon/kathryn-jean-lopez>

Marsh, Charles. <http://cryptome.org/info/wtc-punch/pict1.jpg> 25 mars 2010. consulté le 02 février.

Mendelsohn, Daniel. Si beau, si fragile. Paris : Flammarion, 2011.

Metz, Christian. L'énonciation impersonnelle ou le site du film. Paris : Méridiens Klincksieck, 1991.

Mongin, Olivier. « La société des écrans. Entretien réalisé avec Claudine Haroche et Georges

Vigarello ». Communications - Ecole des Hautes Etudes en Sciences Sociales - Centre d'Etudes transdisciplinaires ( Sociologie, anthropologie, histoire ), $\mathrm{n}^{\circ}$ 75, 2004. 219-227.

Morrissey, Priska. Historiens et cinéastes : rencontre de deux écritures. Paris : l'Harmattan, 2004.

National Institute of Standards and Technology. 8 septembre 2006. Consulté le 12 janvier 2012. <http://www.nist.gov/public_affairs/factsheet/nist_investigation_911.cfm>,

Odin, Roger. La théorie du film. Paris: Albatros, 1980. 
Pollak, Mickael. «A 9/11 survivor », New York Times. 11 septembre 2005. Consulté le 12 janvier 2012.

<http://www.nytimes.com/2005/09/11/nyregion/thecity/11fyi.html? adxnnl=1\&adxnnlx=1328790352-A3Yc+yy7boT9GhgJW69SPg>

Prince, Stephen. Classical Film Violence Designing And Regulating Brutality In Hollywood Cinema 1930 1968. New Brunswick, New Jersey, Londres: Rutgers University Press, 2003.

---. Firestorm: American Film in the Age of Terrorism. New York: Columbia University Press, 2009.

---. History of the American Cinema 10. A New Pot of Gold: Hollywood Under the Electric Rainbow, 1980-1989. New York: Charles Scribner's Sons, 2000.

Samoilova, Gulnara. « Sans titre ». Time, Special Issue, septembre 2001:13-14.

Spielgelman, Art. In the Shadow of No Towers. New York: Viking Adults, 2004.

Surowiecki, David. « Sans titre ». Time, Special Issue, septembre 2001 : 9-10.

Tesson, Charles. « Retour à l'envoyeur ». Cahiers du cinéma, n 561, octobre 2001. 42-44.

Thomas, Cal. «'World Trade Center' is a world class movie ». 20 juillet 2006. Consulté le 09 décembre 2012.

<http://townhall.com/columnists/calthomas/2006/07/20/

world_trade_center_is_a_world_class_movie/page/full/>

Toplin, Robert Brent. Oliver Stone's USA: Film, History and Controversy. Lawrence : University Press of Kansans, 2000.

Vienne, Patrick. «You are here, la ville en plan d'ensemble dans le cinéma américain contemporain ». In La ville au cinéma. Ed. Julie Barillet, Françoise Heitz, Patrick Louguet, Patrick Vienne. Arras : Artois Presses Université, 2005. 137-158.

Virilio, Paul. Ville panique. Ailleurs comme ici. Paris: Galilée, 2004.

Wills, Garry, Reagan's America, New York: Viking Penguin, 1988.

A.I. Artificial Intelligence, dir. Steven Spielberg, Amblin Entertainment, 2001.

Alexander, dir. Oliver Stone, Warner Bros. 2004.

Armageddon, dir. Michael Bay, Touchstones Pictures, 1998.

Deep Impact, dir. Mimi Leder, Paramount/Dreamworks, 1997.

Fight Club, dir. David Fincher, 20th Century Fox, 1999.

Independence Day, dir. Roland Emmerich, 20th Century Fox, 1996.

JFK, dir. Oliver Stone, Warner Bros., 1991.

Macadam Cowboy, dir. John Schlesinger, United Artists, 1969.

Born on the Fourth of July, dir. Oliver Stone, Universal Pictures, 1989.

Nixon, dir. Oliver Stone, Cinergi Pictures, 1995.

Platoon, dir. Oliver Stone, Orion Pictures, 1985.

Traffic in Souls, dir. George Loane Tucker, Universal. 1913.

Natural Born Killers, dir. Oliver Stone, Warner Bros., 1994.

Dog Day Afternoon, dir. Sidney Lumet, Warner Bros., 1975. 
United 93, dir. Paul Greengrass, Universal, 2006.

Wall Street, dir. Oliver Stone, 20th Century Fox, 1987.

Wall Street: Money Never Sleeps, dir. Oliver Stone, 20th Century Fox, 2010.

World Trade Center, dir. Oliver Stone, Paramount, 2006.

\section{NOTES}

1. Quatre mois après Vol 93 (United 93, Paul Greengrass, 2006), centré sur l'acte de résistance présumé des passagers à bord du seul avion à n'avoir pas atteint sa cible.

2. Ne serait-ce que dans les cinq ans précédant les attentats: Independence Day (ID4, Roland Emmerich, 1996), Deep Impact (Mimi Leder, 1997), Armageddon (Michael Bay, 1998), Fight Club (David Fincher, 1999), A.I (Steven Spielberg, 2001).

3. "The movies set the pattern, and these people have copied the movies. Nobody would have thought to commit an atrocity like that unless they'd seen it in a movie. How dare we continue to show this kind of mass destruction in movies. I just believe we created this atmosphere and taught them how to do it »: propos rapporté du cinéaste Robert Altman, Associated Press Wire Story, 17 octobre 2001 (Prince, 2003, 286).

4. «Si [les images du cinéma catastrophe] sont vérifiées et retrouvées dans celle de la destruction du World Trade Center, de manière très troublante, au cadrage près, [...] elles n'ont servi à rien : elles ne nous ont pas préparé à vivre ce qu'on a vu à la télévision. A quoi sert le cinéma s'il ne remplit pas aussi cette tâche?» (Tesson, 43).

5. «En même temps [que l'image] exalte l'événement, elle le prend en otage. [...] L'image consomme l'événement, au sens ou elle l'absorbe et le donne à consommer. Certes elle lui donne un impact inédit jusqu'ici mais en tant qu'événement-image. Qu'en est-il de l'événement réel, si partout l'image, la fiction, le virtuel perfusent dans la réalité ? [...] La réalité dépasse-t-elle vraiment la fiction? Si elle semble le faire, c'est qu'elle en a absorbé l'énergie, et qu'elle est ellemême devenue fiction » (Baudrillard, 2002, 37-39).

6. L'horreur de l'agression qui a pu si vivement transir la population ne provient-elle pas d'abord de ce que la rumeur nous a fourni comme paramètres complétifs au visible et qui, loin de toute caméra, ne peut avoir d'existence en nous qu'imaginaire : la barbarie des assassinats commis à l'intérieur des appareils à l'aide d'ustensiles triviaux transformés en armes blanches? L'idée de cet artisanat de fortune n'éloigne-t-il pas la réussite de l'opération de toute logistique spectaculaire? Par ailleurs, il est encore important de rappeler que le vol United Airlines 175 qui s'est écrasé en second contre le World Trade Center, un quart d'heure après le vol American Airlines 11, en direct devant les caméras de télévision américaine, a initialement décollé de l'aéroport de Boston avec un quart d'heure de retard. Il parait légitime d'envisager que l'objectif terroriste était de heurter les deux tours, non pas l'une après l'autre, mais en même temps. La désynchronisation ayant favorisé la présence médiatique et la visibilité spectaculaire et «cinématographique» de l'opération parait n'avoir été qu'accidentelle. Voir http:// www.historycommons.org/timeline.jsp?

timeline=complete_911_timeline\&day_of_9/11=ua175.=\%3e

7. Clément Chéroux a analysé de nombreuses Unes de journaux internationaux dans les jours qui suivirent les attentats pour montrer que les photographies d'illustration étaient très souvent similaires, constituant un spectre iconographique de l'événement paradoxalement réduit et stéréotypé au vu du nombre de sources photographiques disponibles.

8. Il est né, a grandi et a étudié le cinéma à New York, ville où se déroule quelques-uns de ses films, dont son court-métrage de fin d'études Last Year in Vietnam (1971) et Wall Street (1987). 
9. «Stone surprised everyone by making a film that emphasized heroism and moral uplift and did not include a social or political perspective on the attacks» [notre traduction].

10. Ecole de peintres paysagistes américains du XVIII ${ }^{\mathrm{e}}$ siècle, initiée notamment par Thomas Cole (1801-1848), attachée à restituer avec exaltation la splendeur et l'immensité des paysages naturels américains inédits pour le public européen. Eloge d'une terre sauvage, utopique, promesse de toutes les richesses, dont les larges tableaux de Asher Brown Durand (1796-1886), Frederick Kensett (1816-1872), et plus tard Frederick Edwin Church (1826-1900) ou Albert Bierstadt (1830-1902) tentaient notamment de rendre les dimensions et la luminosité.

11. «Tout mouvement de caméra est en soi une instance d'énonciation » (Odin, p. 131).

12. (Vienne, 137-158)

13. Stone y a souscrit depuis une nouvelle fois dans Wall Street : l'Argent Ne Dort Jamais (Wall Street: Money Never Sleeps, 2010).

14. Stone réemploiera à plusieurs reprises dans son œuvre le procédé des sautes de couleurs au noir et blanc, notamment dans Tueurs Nés (Natural Born Killer, 1994) et Nixon (1995).

15. L'histoire du film est justement centrée sur les malversations financières du cynique magnat de la bourse Gordon Gekko (Michael Douglas) et de son emprise sur son disciple, le naïf Bud Fox (Charlie Sheen).

16. Comme le rappelle David I. Grossvogel au sujet du cinéma romantique de l'âge d'or Hollywoodien : «Manhattan was meant to be a different world, one whose sublime verticality was a metaphor for the city's superiority over others » (Grossvogel, 117).

17. La musique est dite anempathique lorsqu'elle manifeste de l'indifférence à l'égard de l'action plutôt que de l'accompagner émotionellement (Chion, 123).

18. Deux remarques s'imposent pour nuancer le ton caustique employé par Stone dans cet incipit. D'abord, la réalité grise et miséreuse de New York était loin d'être refoulée jusqu'en 1987 du visible cinématographique. Sans avoir à remonter loin : le cinéma américain des années soixante dix s'est longuement attaché à mettre en scène la ville du point de vue des exclus et à défaire l'illusion romantique en faveur d'une représentation plus violente et naturaliste (Midnight Cowboy, John Schlesinger, 1969 ; Dog Day Afternoon, Sidney Lumet, 1975). Peut-être Stone avait-il pour ambition d'enrayer la superficialité, souvent dénoncée, du cinéma hollywoodien des années 80 en convoquant certaines figures filmiques plus largement déconsidérées dans cette décennie ? Ensuite, la critique des milieux de la finance à l'œuvre dans Wall Street n'empêche pas l'expression d'une certaine fascination à son égard de la part d'Oliver Stone. Le film cultive l'ambiguïté en scrutant avec complaisance le mode de vie cynique des tenants du capitalisme et les privilèges rêvés de leur pouvoir. En cela, Wall Street rejoint d'autres films américains des années 80 , tout autant partagés entre critique et fascination du capitalisme (Trading Places, John Landis, 1983 ; Working Girl, Mike Nichols, 1988) (Grossvogel, 134-136).

19. Selon Garry Wills, l'optimisme du président Reagan aurait initié le retour d'une Amérique impérialiste triomphante après les soubresauts sociopolitiques des années soixante-dix, notamment par une glorification utopique de ses valeurs fondatrices. L'idée de l'Amérique telle que Reagan la vante serait « en équilibre entre le Bon Vieux Temps et le Glorieux Monde Actuel. Nous sommes passé d'une perfection à une autre, à travers un interstice auquel il valait mieux ne pas faire allusion ». [ " Poised between the Good Old Days and the Brave New World. We pass from one perfection to another, through an interspace it were best not to advert to »]. Garry Wills, Reagan's America, New York : Viking Penguin, 1988, p. xiii. [Notre traduction].

20. Après la chute du communisme, l'Amérique aurait conquis de façon apparemment définitive un régime démocratique et libéral régnant pacifiquement sur le monde (Fukuyama 1992).

21. A l'inverse, dans United 93, Paul Greengrass instaure dès les premiers plans, avant même le surgissement de l'événement, une mise en scène heurtée, simulant la prise de vue sur le vif, affectée d'une tension encore sans objet défini. 
22. Le plan élégiaque sur la rivière Hudson connaît à la fin du film son envers symétrique : la dense fumée de cendre camoufle au centre du même paysage le cratère noir béant dans lequel les tours ont sombré.

23. " As moviegoers began to see trailers for the new movie United 93 in theaters a few weeks ago, audience members cried "too soon"-some of them literally crying " (Lopez).

24. Dès les premiers temps du cinéma, la représentation de New York assimilait la ville à un espace souvent malfamé sous la surveillance plus ou moins rigoureuse de la police. Dans Traffic in Souls (George Loane Tucker, 1913, Universal), un exemple parmi d'autres, un policier ouvrait l'œil à la sortie de la gare pour surveiller les charlatans.

25. Par ailleurs, sans doute n'est-il pas anodin que l'affiche exposée sur le fronton soit celle du film Zoolander (Ben Stiller, 2001), tourné à New York avant les attentats mais sorti tout de suite après, qui fut l'un des premiers à avoir sciemment biffé le World Trade Center de l'image par manipulation numérique pour a-temporaliser la diégèse après la catastrophe.

26. En suivant la définition d'André Gardies, le diégétique concerne «le problème de l'énonciation narrative [...] et les divers types de niveaux et de relations de personne qu'un narrateur peut entretenir avec ce qu'il raconte ». Le filmique quant à lui se rapporte à " tout ce qui apparaît dans un film », qui est rendu visible et participe à l'esthétique du film (Bessalel et Gardies, 59 et 89 ).

27. Leur présence dans la presse s'est raréfiée au fil des jours, pourrions-nous nuancer. Ainsi, dans un numéro spécial paru le lendemain des attentats, le magazine Time publie en double page un cliché de très grand format pris par David Surowiecki, peu reproduit depuis, présentant trois corps tombant les uns au-dessus des autres sur fond de ciel bleu : Time (septembre 2001, 9-10).

28. Notamment en Une d'un quotidien de Pennsylvannie, The Herald (12 septembre 2001, 1), et dans le New York Times (12 septembre 2001, A7).

29. Exposé au Rockefeller Center en 2002, avant que de nombreuses plaintes n'obligent l'auteur à retirer son œuvre.

30. « Tout ce que je peux vous dire, raconte James Whitney à notre correspondante Afsané Bassir Pour, c'est que je ne pourrai plus jamais fermer les yeux sans voir les virgules noires tombant du ciel, ces corps courbés comme des virgules noires sautant par douzaines des fenêtres du bâtiment, face à mon loft », (George, 2001).

31. <https://sites.google.com/site/wtc7lies/7-69_landing-gear-tire_west-rector-s-full.jpg

32. National Institute of Standards and Technology

<http://www.nist.gov/public_affairs/factsheet/nist_investigation_911.cfm

33. La même femme en rouge est également présente sur un cliché de Gulnara Samoilova, publié en double page dans le magasine Time (Samoilova, 13-14).

34. «It is one of the greatest pro-American, pro-family, pro-faith, pro-male, flag-waving, God Bless America films you will ever see » (Thomas). « The lack of any sustained political discourse beyond the most conventional of feel-good American patriotism makes this film one of director Oliver Stone's least impressive works (Cettl, 290).

35. "Without going outside, and beyond, the points of view of the main characters, without taking the larger view, by staying inside his microcosmic framework he cannot create a dialogue among the perspectives raised by the events that the film portrays » [notre traduction]. 


\section{RÉSUMÉS}

Cet article analyse comment le film World Trade Center d'oliver Stone met en scène les attentats du 11 septembre 2001. À travers l'analyse de quatre séquences situées dans les vingt premières minutes du film, il s'agira de montrer comment la représentation médiatique a pu octroyer au 11/9 une identité visuelle préétablie auprès de laquelle toute reconstitution filmique semble contrainte de se positionner. Mais la transposition de l'événement en fiction cinématographique doit-elle nécessairement passer par l'adaptation de ses images? Quels écarts Oliver Stone essaiet-il d'établir avec les canons médiatiques sur lesquels reposent la mémoire collective ? De quelle singularité, de quelle autonomie formelle World Trade Center peut-il se targuer?

The purpose of this paper is to analyse the aesthetic implications of filmic adaptations of the terrorist attacks of September 11, 2001. It considers how Hollywood cinema make the spectator believe in the authenticity of the disaster, while examining the use of pre-existing visual images of the event. Finally, it discusses the extent of formal autonomy in the filmic reconstruction of the event.

\section{INDEX}

Keywords : September 11 2001, World Trade Center, New York, Oliver Stone, cinema, Hollywood, aesthetic, representation, disaster, spectacular, visible, unwatchable, event, ethical

Mots-clés : 11 septembre 2001, catastrophe, World Trade Center, New York, cinéma, esthétique, éthique, événement, Hollywood, inmontrable, représentation, spectaculaire, visible

\section{AUTEURS}

\section{VINCENT SOULADIÉ}

Laboratoire de Recherche en Audiovisuel (LARA) Université Toulouse II le Mirail Doctorant en études filmiques, Attaché Temporaire d'Enseignement et de Recherche vsouladi@univ-tlse2.fr 